África (São Paulo, 1978, Online), São Paulo, n. 42, p. 156-176, 2021

http://dx.doi.org/

\title{
Les abordages théoriques sur les défis du développement des pays africains et les leçons qu'on peut en tirer.
}

Georges Nzongola-Ntalaja ${ }^{2}$

Resumé: Depuis les indépendances africaines, plusieurs théories ont été formulées sur les défis du développement en Afrique et les voies les plus appropriées pour éradiquer la pauvreté et améliorer le niveau de vie des masses populaires. Malgré leurs incarnations multiples, deux courants théoriques contradictoires continuent d'animer le débat sur ce sujet. Il s'agit du courant hégémonique véhiculé par les pays occidentaux et les institutions financières internationales sous leur contrôle, et le courant contre-hégémonique des progressistes africains. Quelles leçons pouvons-nous tirer de ces deux approches contradictoires sur les défis du développement en Afrique?

Mot-clés: Développement des pays africains; Politique africaine

Sumário: Desde as independências africanas, várias teorias foram formuladas sobre os desafios do desenvolvimento na África e as formas mais adequadas de erradicar a pobreza e melhorar o padrão de vida das massas populares. Apesar de suas encarnações múltiplas, duas correntes teóricas contraditórias continuam a animar o debate sobre o assunto. Uma é a corrente hegemônica

Communication au Séminaire International sur la Recherche et l'Enseignement à l’Université Africaine dans le Contexte de la Globalisation et l'Interface avec le Brésil: Perspectives Epistémologiques Emergentes, Nouveaux Horizons Thématiques et Défis, tenu à l'Université de São Paolo, Brésil, du 3-5 septembre 2012. 
GEORGES NZONGOLA-NTALAJA Les abordages théoriques sur les défis du développement des pays africains et les leçons qu'on peut en tirer

defendida pelos países ocidentais e pelas instituições financeiras internacionais sob seu controle, e a contra-corrente levada a efeito pelos progressistas hegemônicos africanos. Que lições podemos tirar dessas duas abordagens contraditórias para os desafios do desenvolvimento na África?

Palavras-chave: Desenvolvimento dos países africanos; Política africana

INTRODUCTION

J'ai l'insigne honneur de clôturer ce séminaire international, organisé en hommage au professeur Fernando Augusto Albuquerque Mourão, directeur fondateur du Centre d'Etudes Africaines (CEA) de l'Université de São Paolo. Mes remerciements les plus sincères aux professeurs Kabengele Munanga et Tania Macedo, Directrice du CEA, pour m'avoir associé à cette rencontre.

Bien que très complexe, le sujet que le Comité Scientifique du séminaire a proposé pour ma communication est à la fois pertinent et d'actualité, dans la mesure où il est au centre des débats sur le devenir de l'Afrique depuis 1960. En 1985, dans un article perspicace publié dans le magazine londonien West Africa, le politologue nigérian Claude Ake avait posé la question de savoir "pourquoi l'Afrique ne se développe-t-elle pas?". ${ }^{3}$ Vingt-sept ans plus tard, cette question continue à nous interpeller, malgré les impressionnantes statistiques de croissance économique annuelle de 5 à 10 pourcent à travers le continent. Aussi encourageantes que ces statistiques puissent paraître, il n'en reste pas moins que la qualité de vie de la grande majorité de la population africaine d'un milliard d'âmes ne fait que se détériorer. Plus que la moitié de cette population continue de vivre en dessous du seuil de pauvreté, soit 2 \$US par jour, tandis que 39\% des Africains vivaient avec moins d'un dollar et 25 centimes par jour en monnaie américaine en $2011 .{ }^{4} \mathrm{Au}$ courant de la même année, 36 des 46 États ayant le niveau le plus bas dans l'Indice de développement humain du Programme des Nations unies pour le développement (PNUD) sont africains. 12 États

\footnotetext{
Claude Ake, "Why Africa is not developing”, West Africa, 17 June 1985.

$4 \quad$ Groupe de la Banque Africaine de Développement, Revue annuelle sur l'efficacité du développement, Édition 2012, BAD, Tunis, p. 14. Le chiffre de 1,25 \$US représente le seuil de pauvreté extrême.
} 
africains figurent dans le groupe de développement humain moyen, quatre dans celui de développement humain élevé, et aucun État africain ne se trouve dans le groupe de développement humain très élevé. ${ }^{5}$

Il est donc évident qu'il ne faut pas confondre la croissance économique avec le développement humain, celui-ci n'étant pas mesuré par le produit national brut par habitant, mais suivant une formule comprenant des indicateurs de la santé (l'espérance de vie notamment), la scolarité et le revenu. Il s'ensuit que l'amélioration du standard de vie du citoyen moyen ne résulte pas automatiquement de la croissance économique, mais de la façon dont cette croissance est répartie au bénéfice de toute la population par la création des emplois, la construction des infrastructures, et la provision des services sociaux. Depuis les indépendances africaines, plusieurs théories ont été formulées sur les défis du développement en Afrique et les voies les plus appropriées pour éradiquer la pauvreté et améliorer le niveau de vie des masses populaires. Malgré leurs incarnations multiples, deux courants théoriques contradictoires continuent d'animer le débat sur ce sujet. Il s'agit du courant hégémonique véhiculé par les pays occidentaux et les institutions financières internationales sous leur contrôle, et le courant contre-hégémonique des progressistes africains. Quelles leçons pouvons-nous tirer de ces deux approches contradictoires sur les défis du développement en Afrique?

Dans son intégration au système mondial actuel, l'Afrique fait figure de continent en crise, une crise multidimensionnelle dont la faillite de l'État est la clé de voûte. Malgré l'indépendance acquise, bon nombre d'États africains sont restés fidèles aux intérêts de la métropole, plus enclins à répondre aux exigences des grandes puissances mondiales et des institutions financières internationales qu'à satisfaire les aspirations de leur peuple pour le bien-être, la démocratie et la souveraineté. Une telle situation ne peut être qu'un foyer de pauvreté, de conflits et des violences: guerres ethniques, rébellions et mutineries, insurrections populaires, luttes pour le contrôle des ressources, etc. Face à cette situation, il est temps, pour les mouvements sociaux africains, de dépasser la notion de l'État héritée de la colonisation, c'est-à-dire de restructurer et réhabiliter le pouvoir de l'État dans ses dimensions redistributives et démocratiques. La réussite d'une telle initiative demande une lecture attentive

\footnotetext{
Programme des Nations Unies pour le Développement, Rapport sur le développement humain 2011, PNUD, New York.
} 
GEORGES NZONGOLA-NTALAJA Les abordages théoriques sur les défis du développement des pays africains et les leçons qu'on peut en tirer

de l'histoire des luttes populaires pour la démocratie et le progrès social, et surtout une mise en pratique des valeurs instigatrices de ses luttes: la recherche de l'unité et de l'intégration nationales, la souveraineté, la lutte contre la dictature et la corruption.

\section{LE COURANT HÉGÉMONIQUE DE LA PENSÉE UNIQUE NÉOLIBÉRALE}

En tant qu'une approche intellectuelle à l'étude du développement économique dans les sociétés non occidentales d'Afrique, d'Asie et d'Amérique latine, le courant hégémonique se manifesta à la suite de la deuxième guerre mondiale comme un discours idéologique reflétant la confrontation entre les camps occidental et soviétique. Dans ce contexte de la guerre froide au niveau international et de la décolonisation dans les anciennes possessions coloniales de l'Europe occidentale en Asie, en Afrique et dans les Caraïbes, l'analyse du développement et du sous-développement dans les sciences sociales fut dominée par les chercheurs occidentaux, dont la vision de développement était synonyme de l'occidentalisation. Contesté par les chercheurs latino-américains, dont la théorie de la dépendance mit en évidence le caractère idéologique du courant hégémonique, celui-ci se révélera comme étant soucieux avant tout de préserver la mainmise occidentale sur les économies du tiers monde. Avec l'effondrement de l'Union soviétique et la fin de la guerre froide, les cercles dominants du capitalisme mondial se sentirent si victorieux et arrogants qu'ils proclamèrent la fin de l'histoire et la canonisation du néolibéralisme comme la pensée unique. ${ }^{6}$ Baptisé sous l'appellation de "Consensus de Washington", le courant hégémonique imposera des politiques d'ajustement structurel (PAS) en Afrique, mais celles-ci échouèrent partout pour être remplacées en 1999 par les documents stratégiques de réduction de la pauvreté (DSRP). Au lieu du consensus de Washington, les puissances occidentales s'efforcent à présent de créer un consensus néolibéral mondial à travers les forums tels que le G-20 et Davos, afin d'associer les bourgeoisies d'État des pays africains et du Sud à une gestion partagée de l'économie mondiale qui, en dernière analyse, restera favorable aux intérêts occidentaux.

6 Lire Ignacio Ramonet, “La pensée unique”, Le Monde Diplomatique, janvier 1995. 
África (São Paulo, 1978, Online), São Paulo, n. 42, p. 156-176, 2021

\section{Croissance Économique par le Changement social, 1960-1969}

Tout comme le développement économique des pays du tiers monde était subordonné à la reconstruction des économies industrielles de l'Europe et du Japon après la deuxième guerre mondiale, les théories de développement et les stratégies d'assistance économiques depuis 1945 constituent des tentatives des pays occidentaux et des organisations internationales sous leur contrôle de gérer l'économie mondiale et le changement dans les pays du Sud. Le but n'est pas d'éradiquer la pauvreté et de réduire des inégalités entre pays riches et pauvres, ou encore moins entre les riches et les pauvres dans les pays du Sud. Si cela était le but recherché, cinquante ans n'auraient-ils pas suffi de marquer ne fut-ce qu'une petite amélioration dans la vie des pauvres d'Afrique?

Dans un excellent survol des politiques de développement occidentales entre 1950 et 1990 dans son livre Development Projects as Policy Experiments: An Adaptive Approach to Development Administration, Dennis Rondinelli montre que l'échec vis-à-vis de la lutte contre la pauvreté est fonction d'au moins trois causes. ${ }^{7}$ La première est que, compte tenu de leur préoccupation avec l'accélération de la croissance économique, les théories de développement conventionnelles sont trop éloignées des réalités complexes des pays du Sud pour réaliser les buts escomptés. La deuxième cause de l'échec est que les stratégies d'assistance au développement associées à ces théories n'ont pas de bénéfices durables par rapport au renforcement des capacités institutionnelles des pays du Sud. Et la troisième est que les méthodes de planning et de mise en œuvre des politiques et stratégies de développement étaient trop rationalistes et partant, inappropriées dans le contexte de complexité et d'incertitude du processus de développement, qui exige une approche beaucoup plus adaptable et comprenant l'innovation, l'expérimentation, l'aptitude de réagir aux pressions sociales, et la capacité de tirer des leçons de l'action en cours.

Symboliquement, l'année 1960 est très importante à plus d'un titre. Baptisée "l'année de l'Afrique" à cause de l'accession à l'indépendance de 17 pays africains, elle est aussi la première année de la "décennie du développement", ainsi proclamée par les Nations unies en janvier 1961. Cette première

\footnotetext{
Dennis Rondinelli, Development projects as policy experiments: An adaptive approach to development administration, $2^{\mathrm{e}}$ ed., Routledge, London et New York, 1993.
} 
GEORGES NZONGOLA-NTALAJA Les abordages théoriques sur les défis du développement des pays africains et les leçons qu'on peut en tirer

décennie du développement (1960-1969) marquera l'abandon de la première stratégie de développement issue de la théorie dominante du développement, la soi-disant "trickle down theory", ou la notion selon laquelle une forte croissance économique finit par profiter à tout le monde, aux riches d'abord mais aussi aux plus démunis ensuite. Cette notion était la religion de la plupart des théoriciens du développement des années 1950, tout comme pour l'extrême droite aux États-Unis aujourd'hui. À cette fausse prémisse que l'accélération de la croissance résultera automatiquement en un meilleur standard de vie pour tous, succéda l'idée que le changement social était indispensable aux processus de croissance et de développement économiques, vu le fait que les problèmes sociaux, économiques et politiques sont inextricablement liés. La nouvelle stratégie, que Rondinelli désigne sous le titre de "croissance économique par le changement social", était basée sur l'idée d'éliminer des obstacles à la croissance par la redistribution des biens de production, le développement des ressources humaines, le contrôle des naissances, et le renforcement des capacités productives dans les secteurs les plus faibles de l'économie. ${ }^{8}$

En théorie, cette approche était prometteuse, tant elle montrait que pour combattre la pauvreté, la redistribution des ressources et l'élimination des pratiques sociales et culturelles qui freinaient le développement étaient indispensables. En pratique, cependant, il n'était pas facile de s'attaquer à ces obstacles dans le contexte de la guerre froide. Malgré les belles paroles dans ce sens par les officiels des gouvernements occidentaux et des agences multilatérales, qui ne manquaient pas de proclamer haut et fort leur profession de foi dans la nécessité du changement social, l'administration du développement s'occupa plus de la réforme que de la révolution. Car tout changement susceptible de déstabiliser des régimes pro-occidentaux et anti-communistes ne pouvait pas être entrepris. Même au niveau des recherches universitaires en Amérique du Nord, la tendance majoritaire était de considérer l'administration du développement comme un outil de gestion des crises ("crisis management") et non de transformation. En définitive, comme l'ancienne stratégie de "trickle down", celle de croissance économique par le changement social échoua misérablement dans sa tentative de réduire la pauvreté en Afrique et dans le tiers monde. Cet échec consacrait aussi l'échec de la "décennie du

$8 \quad$ Rondinelli, p. 28. 
África (São Paulo, 1978, Online), São Paulo, n. 42, p. 156-176, 2021

développement", d'où la nécessité d'une "deuxième décennie du développement", de 1970 à 1979.

\section{Croissance ÉCONomique AVec ÉQuité sociale}

Une nouvelle stratégie de développement fut adoptée pour cette décennie, focalisée sur la réduction ou "l'atténuation" de la pauvreté. Cette stratégie est mieux capturée dans la loi d'assistance étrangère de 1973 adoptée par le Congrès américain, qui circonscrit le mandat public à privilégier les personnes les plus pauvres dans l'octroi de l'assistance économique. C'est dans cette perspective de l'orientation de l'assistance économique à la résolution de problèmes auxquels les gens les plus pauvres font face que Rondinelli désigne cette stratégie comme étant celle de "la croissance économique avec équité sociale".

La stratégie se manifestera à travers trois approches majeures: 1) le développement rural intégré; 2) l'assistance aux groupes particuliers, en occurrence, les femmes, les enfants, les travailleurs agricoles, etc.; et 3) les besoins humains fondamentaux (BHF). En tant que l'approche la mieux connue et la plus populaire de cette stratégie, celle de BHF consistait de deux volets: d'une part, des exigences minimales de consommation familiale, incluant la nourriture, le logement, et l'habillement; et, d'autre part, les services communautaires essentiels, tels que l'éducation de base, les soins de santé primaires, l'eau et l'hygiène, et la recréation. Chacune de ces deux composantes de BHF requiert l'augmentation de la productivité et du revenu des pauvres comme un droit humain fondamental pour leur permettre d'améliorer leur standard de vie. En même temps, une main d'œuvre en bonne santé, bien nourrie, et mieux instruite devrait disposer des compétences, force et motivation nécessaires pour augmenter la production de biens et services, ainsi que le pouvoir d'achat requis pour avoir accès aux biens et services en tant que consommateurs.

Chacune de trois approches de la stratégie de croissance économique avec équité sociale a échoué à atteindre ses objectifs. Le développement rural intégré ne pouvait pas réussir dans l'absence d'une résolution équitable du problème de la propriété foncière, particulièrement là où certains groupes 
GEORGES NZONGOLA-NTALAJA Les abordages théoriques sur les défis du développement des pays africains et les leçons qu'on peut en tirer

n'avaient pas de droits fonciers, ainsi que d'un investissement considérable dans l'infrastructure de production et celle des services sociaux en milieu rural. L'assistance aux groupes particuliers, une approche que la Division des dimensions sociales d'ajustement de la Banque mondiale développera pour le bénéfice des "groupes vulnérables" afin de leur assurer des moyens d'existence décents, devait échouer à cause de l'insuffisance des moyens financiers consacrés à cette cause et de l'absence de la démocratie. Ne disposant pas des droits démocratiques, les pauvres n'avaient aucune voix dans l'élaboration des politiques de développement et la répartition des fonds publics nécessaires pour leur mise en œuvre.

Ces raisons s'appliquent aussi à l'échec du développement rural intégré et des besoins humains fondamentaux. Dans ce dernier cas, l'approche BHF eut l'avantage d'avoir un grand "champion" en la personne de Robert $\mathrm{McNa}$ mara, président de la Banque mondiale entre 1968 et 1981. Une campagne publicitaire bien financée avec des brochures de luxe montrait jusqu'où l'ancien Secrétaire à la défense américain était préparé d'aller en battant tambour pour les BHF. Malgré ses efforts, l'échec de cette approche était inévitable. Car il s'agissait d'un palliatif à la place d'une sérieuse tentative d'éradiquer la pauvreté. McNamara et la Banque cherchèrent de mettre fin à la pauvreté sans toutefois s'attaquer aux structures mêmes responsables pour sa création et sa reproduction aux niveaux local, national et international. Ces structures comprennent l'inégalité dans la propriété foncière, la dénégation de droits humains fondamentaux, le manque de voix dans l'élaboration des politiques publiques, et l'absence d'une situation équitable dans les relations économiques internationales.

\section{Auustement structurel et le consensus de Washington (1980-99)}

Comme la plupart des pays du Sud se sont plongés dans une crise économique sévère pendant la deuxième moitié des années 1970, les institutions de Bretton Woods adoptèrent une nouvelle et rigoureuse politique économique appelée "programmes d'ajustement structurel" (PAS). Comme stratégie de développement dominante pour les années 1980 et 1990, les PAS devinrent pour l'Occident la réponse la plus appropriée à la crise économique 
África (São Paulo, 1978, Online), São Paulo, n. 42, p. 156-176, 2021

africaine, caractérisée par une baisse croissante de production, réductions considérables de recettes d'exportation, l'augmentation de la dette extérieure, et la réduction du pouvoir d'achat de la majorité de la population. Comme tous ces éléments de la crise étaient perçus comme la faute de l'État et de l'expansion de son rôle dans l'économie, la clé de voûte des PAS consiste en un changement radical de l'État au marché comme le mécanisme principal de la répartition des ressources. Cela implique un rôle réduit pour l'État dans l'économie par la privatisation des entreprises publiques et une réduction draconienne du budget et du nombre des fonctionnaires. Par ce biais, les biens publics furent vendus au rabais et les dépenses publiques pour les services sociaux de base sérieusement réduites.

Les PAS furent l'aboutissement logique de la domination de la théorie et la pratique du développement par ce qui deviendra connu plus tard comme le "consensus de Washington". Il s'agit d'un ensemble de mesures qui étaient censées conduire les pays du Sud à la prospérité. Sous l'impulsion des politiques économiques de Ronald Reagan aux Etats-Unis et Margaret Thatcher en Grande Bretagne, ces mesures comprenaient toute la gamme de politiques néolibérales et de marché libre incluant l'austérité fiscale et monétaire, la baisse d'impôts, la suppression des subsides étatiques, la libéralisation des taux d'intérêts et du commerce extérieur, la réduction des taux d'échange, la dérégulation, la privatisation, et la promotion d'investissements étrangers directs. Le consensus de Washington se résume donc par la mise en place d'une nouvelle orthodoxie néolibérale, une "pensée unique" apparemment valable et utile pour tous les pays du monde.

Ironiquement, une des conditions requises pour l'obtention des prêts d'ajustement structurel auprès du Fonds monétaire international (FMI) et de la Banque mondiale était la pratique de la "bonne gouvernance". Au début, compte tenu de la répugnance de ces institutions de s'ingérer dans les affaires politiques des alliés de l'Occident dans les pays du Sud, cette exigence était conçue en termes de l'efficacité et la transparence de la gestion de la chose publique, ainsi que de la compétence de l'appareil judiciaire à régler les problèmes ayant trait aux contrats avec les multinationales. Avec la chute des régimes staliniens en Europe de l'Est et la désintégration de l'Union soviétique, la voie s'est ouverte pour tout un train de mesures de réforme démocratique comme conditions préalables à l'obtention d'assistance économique. L'ironie 
GEORGES NZONGOLA-NTALAJA Les abordages théoriques sur les défis du développement des pays africains et les leçons qu'on peut en tirer

ressort du fait que des mesures de réforme économique conçues à Washington sont imposées aux dirigeants africains qui, à leur tour, doivent les faire avaler à leurs citoyens sans aucun débat. La quête de la gouvernance démocratique restait ainsi insaisissable dans la mesure où elle ne pouvait pas se manifester dans le cadre des structures et des traditions autoritaires qui ne reconnaissent pas aux acteurs nationaux le droit d'initier ou même de prendre part aux réformes économiques par le truchement des institutions et procédures démocratiques. ${ }^{9}$ En d'autres termes, l'ajustement structurel et la démocratie ne pouvaient pas être compatibles. En fin de compte, les PAS et d'autres prescriptions du consensus de Washington n'ont pas réussi à augmenter la richesse et la prospérité en Afrique.

\section{DoCumentS StRATÉGIQUeS de RÉdUCtION DE LA PAUVRETÉ}

En 1999, après quarante ans d'échec dans la lutte contre la pauvreté, le FMI et la Banque mondiale inaugurèrent une fois de plus une nouvelle stratégie pour réduire la pauvreté. Conçu comme toujours dans le cadre du courant hégémonique, cette stratégie est innovatrice dans ce sens que le schéma directeur venant de Washington est susceptible de modification sur le terrain par l'apport du pays bénéficiaire, qui assume la paternité ou la possession de son propre "document stratégique de réduction de la pauvreté". Le document en question contient un sommaire du cadre macroéconomique du pays, la description de ses politiques tant structurelles que sociales, un aperçu des programmes conçus pour promouvoir la croissance et réduire la pauvreté, ainsi que les exigences de financement externe pour l'ensemble du programme. Il s'agit, en principe, d'un plan économique de trois ans élaboré par le pays membre avec l'assistance technique des équipes du FMI et de la Banque mondiale, et en collaboration avec plusieurs parties prenantes, notamment la société civile et d'autres partenaires au développement.

Si ce document de stratégie est censé être la "propriété" du pays membre qui en revendique la paternité, il n'en reste pas moins que le DSRP reflète les priorités de développement externes, d'autant plus que son finan-

\footnotetext{
9 Lire Adebayo Olukoshi, The Elusive Prince of Denmark: Structural adjustment and the crisis of governance in Africa, Nordic Africa Institute, Uppsala, 1998.
} 
África (São Paulo, 1978, Online), São Paulo, n. 42, p. 156-176, 2021

cement par des sources externes est indispensable pour sa mise en œuvre. Or ce financement, inutile de le souligner, provient des schémas préconçus des organisations multilatérales et des agences de développement des pays occidentaux, aujourd'hui dominées par la pensée néolibérale. En outre, il est curieux de constater que plus de cinquante ans après le lancement de la première décennie du développement, les représentants du courant hégémonique continuent de nous endormir en nous parlant de leur volonté de réduire la pauvreté. Il y a suffisamment des ressources alimentaires et financières au monde pour éradiquer la pauvreté et garantir à chaque être humain le minimum d'une vie décente. Ce n'est que la volonté politique au sein des gouvernements et la mobilisation politique au sein des masses populaires qui manquent pour assurer une équitable redistribution de ces ressources à travers le monde entier.

\section{LES OBJeCtifS DU MILLÉNAIRE POUR LE DÉVELOPPEMENT (OMD)}

L'échec des pays du Sud et de la communauté internationale d'éradiquer la pauvreté, la faim et les maladies qui sont faciles à prévenir dans un monde riche en ressources naturelles, matérielles et humaines a été une raison majeure pour la tenue en septembre 2000 au quartier général de l'ONU à New York du Sommet du Millénaire pour débattre de ce problème. La déclaration adoptée par ce sommet mit en place une nouvelle stratégie de développement, les Objectifs du Millénaire pour le Développement (OMD). Malgré le fait que les idées-clé des OMD sont basées sur les stratégies occidentales précédentes, la nouvelle stratégie a l'avantage d'avoir été adoptée de manière consensuelle par la communauté internationale dans son ensemble. Encore timides comme objectifs dans la lutte contre la pauvreté, cette stratégie marque un pas en avant en proposant huit objectifs à atteindre, surtout en 2015 au plus tard, ensemble avec les cibles à réaliser pour chaque objectif ainsi que des indicateurs pour ces cibles. Les huit objectifs sont les suivants:

1. Eradiquer la pauvreté et la faim extrêmes

2. Réaliser l'éducation primaire universelle

3. Promouvoir l'égalité des sexes et responsabiliser les femmes 
GEORGES NZONGOLA-NTALAJA Les abordages théoriques sur les défis du développement des pays africains et les leçons qu'on peut en tirer

4. Réduire la mortalité infantile

5. Améliorer la santé maternelle

6. Combattre VIH/SIDA, malaria, et autres maladies

7. Assurer la pérennité de l'environnement

8. Développer un partenariat global pour le développement

Les OMD constituent un engagement au niveau mondial. L'OMD-8, qui exige la mise en place d'un partenariat global pour le développement, est un contrat entre les pays développés et les pays du Sud, qui doivent respecter leurs obligations respectives pour le succès de cette stratégie de développement. La responsabilité primaire des pays du Sud est d'œuvrer pour la réalisation de sept premiers objectifs par la gouvernance démocratique, l'utilisation efficiente des ressources, et leur disponibilité d'être redevables envers leurs citoyens. Pour que cela réussisse, les pays développés doivent tenir leurs engagements dans le partenariat, par la provision d'une assistance économique plus effective, un allégement de la dette extérieure des pays du Sud, et de conditions d'échange équitables.

Malheureusement, les OMD donnent l'impression du déjà vu, tant ils ressemblent aux BHF des années 1970. Le monde a-t-il suffisamment changé depuis lors pour permettre un véritable assaut contre la pauvreté dans le cadre de la globalisation? Les pays africains sont-ils capables de s'approprier les $\mathrm{OMD}$ et de les réaliser avec succès ?

\section{LE COURANT CONTRE-HÉGÉMONIQUE DES PROGRESSISTES AFRICAINS}

Pendant les cinq décennies de l'expérimentation occidentale dans la théorie et la pratique du développement pour les pays du Sud, ceux-ci ne sont pas restés les bras croisés. Leurs intellectuels ont à leur tour développer leurs propres perspectives sur la place du tiers monde dans l'économie mondiale, des modèles de société alternatifs, ainsi que les stratégies de développement les plus appropriées pour réaliser ces visions. Parmi les différents apports 
au courant contre-hégémonique sur les défis du développement en Afrique, trois sources de réflexion et d'inspiration doivent retenir notre attention. Il s'agit du travail des commissions économiques régionales des Nations unies, et plus particulièrement la Commission économique des Nations unies pour l'Afrique (CEA); le projet panafricaniste d'autodétermination, d'indépendance économique et de solidarité africaine tel qu'il fut exprimé par les dirigeants progressistes de la lutte pour l'indépendance; et les leçons à tirer des conférences nationales souveraines des années 1990 sur la restructuration de l'Etat afin de lui permettre de satisfaire les aspirations profondes du peuple pour la démocratie et le progrès social.

\section{Leçons tirées de la Commission Economique des Nations Unies pour L'Afrique (CEA)}

Dès les années 1950, les commissions économiques de l'ONU en tant que think tanks pour leurs continents respectifs jouèrent un rôle important dans la formulation des stratégies de développement économiques. En ce qui concerne la critique du courant hégémonique, l'initiative a été prise par la Commission économique pour l'Amérique latine (ECLA), dont le rôle de fer de lance à cet égard est universellement reconnu. Un des éclairages les plus importants de l'ECLA fut de démontrer qu'au lieu d'être un état naturel de toutes les sociétés, le sous-développement est une réalité historiquement construite de telle manière que le sous-développement de la périphérie du système capitaliste mondial était la condition sine qua non du développement au centre. Une des leçons tirées de ce constat fut de ne plus compter sur l'exportation des matières premières, dont les prix ne faisaient que chuter sur le marché mondial, et de réduire en revanche la dépendance vis-à-vis des pays développés en adoptant une stratégie de développement autonome.

Inspirée par l'exemple de l'ECLA, la Commission économique des Nations unies pour l'Afrique (CEA) a, depuis sa mise en place en 1958, fait le plaidoyer en faveur de la coopération et l'intégration régionales. En collaboration avec la Banque africaine de développement (BAD) et le Secrétariat de l'Organisation de l'Unité africaine (OUA), la CEA réussit à convaincre les Chefs d'État et de gouvernement africains d'endosser en 1980 
GEORGES NZONGOLA-NTALAJA Les abordages théoriques sur les défis du développement des pays africains et les leçons qu'on peut en tirer

le plan le plus compréhensif de croissance autonome et de développement intégré que le continent africain ait jamais conçu, le Plan d'Action de Lagos pour la mise en ouvre de la stratégie de Monrovia pour le développement économique de l'Afrique (PAL). Le PAL représente une stratégie intégrée de développement par les améliorations dans les secteurs de l'industrie, transports et communications, développement des ressources humaines et naturelles, science et technologie, avec un but ultime d'intégration économique au niveau continental.

En dépit de quelques progrès réalisés dans la création des communautés économiques régionales comme composantes de base vers cette intégration continentale dans le cadre du Traité d'Abuja (1991) sur la communauté économique africaine, le PLA n'a pas eu les effets escomptés. Trois raisons majeures peuvent être avancées pour expliquer cette défaillance: 1) l'inaction africaine par rapport aux ressources budgétaires nécessaires à l'exécution du plan et l'élimination des contraintes bureaucratiques sur la libre circulation des personnes et des biens; 2) les contraintes structurelles dues au fait que les économies africaines sont avant tout orientées vers l'exportation des matières premières, ce qui fait que le commerce inter-Afrique représente encore un infime pourcentage des statistiques commerciales nationales; et 3) l'hostilité des institutions de Bretton Woods, bien illustrée par la réponse de la Banque mondiale en 1981 au PAL avec son propre plan pour le développement de l'Afrique, Développement accéléré en Afrique sub-saharienne: un programme d'action. Mieux connu sous le titre de Rapport Berg (du nom du professeur Elliot Berg, coordonnateur du groupe d'experts qui avait rédigé le rapport), le plan de la Banque propose une économie basée sur l'agriculture et orientée vers l'exportation. Il s'agit là d'une vision de l'avenir de l'Afrique qui est diamétralement opposée à celle du PAL et sert de travail de sape vis-à-vis de ce dernier.

Une autre contribution majeure de la CEA à la critique du courant hégémonique de la théorie du développement fut son opposition aux PAS. Sous la direction du professeur Adebayo Adedeji, Secrétaire exécutif entre 1978 et 1991, la Commission mena une lutte vigoureuse contre les PAS et leur coût social négatif en Afrique. En 1989, par exemple, la CEA publia son alternative aux PAS, intitulée African Alternative Framework to Structural Adjustment Programs for Socio-Economic Recovery and Transformation 
(AAF-SAP). La position de la CEA sur ce point reflétait le point de vue de la majorité d'intellectuels africains, pour qui l'ajustement décrété à Washington non seulement créait de problèmes de légitimité pour les États africains, mais encourageait aussi ces derniers à renforcer leurs tendances dictatoriales et à compter sur la répression pour imposer contre la volonté du peuple des mesures économiques impopulaires. ${ }^{10}$ Cinq organisations méritent d'être reconnues pour leur contribution à la critique du courant hégémonique et pour leur soutien à la CEA pendant cette période. Il s'agit de 1) l'Institut africain pour le développement économique et le planning (IDEP) sous la direction de Samir Amin entre 1970 et 1980;2) le Conseil pour le développement de la recherche en sciences sociales (CODESRIA); 3) l'Association africaine de science politique (AASP); 4) l'Institut pour les alternatives africaines (IFAA), dont la réponse au FMI et à la Banque mondiale fut publiée en plusieurs ouvrages sous la direction de Bade Onimode"; et 5) le Forum du Tiers Monde (FTM), avec une contribution majeure de Samir Amin à la pensée alternative. ${ }^{12}$

Entre 1991 et 2012, la Commission semble avoir abandonné cet engagement panafricaniste pour se rallier au libéralisme de Thabo Mbeki et Abdoulaye Wade, deux des initiateurs du Nouveau Partenariat pour le Développement de l'Afrique (NEPAD), un projet qui fut entériné par le G-8 en 2002 à Kananaskis, au Canada, avant qu'il ne soit porté à la connaissance du public africain! Carlos Lopes, un brillant intellectuel et membre de plusieurs réseaux scientifiques africains, vient d'accéder au poste de Secrétaire exécutif de la CEA. J'ose croise, et espérer, que sous sa direction, la Commission renouera avec son passé de critique du courant hégémonique et d'avocat d'une pensée alternative favorable aux intérêts des peuples d'Afrique.

\footnotetext{
10 Lire Bonny Ibhawoh, "Structural adjustment, authoritarianism and human rights in Africa," Comparative Studies of South Asia, Africa and the Middle East, Vol. XIX, nº 1 (1999), p. 158-167.

11 The IMF, the World Bank, and the African Debt, Zed Books, London et Atlantic Highlands, NJ, 1989, 2 vols.; Alternative development strategies for Africa, Institute for African Alternatives, London, 199091, 2 vols.; The African response: Adjustment or transformation, Institute for African Alternatives, London, 1992.

12 Lire Pensée sociale critique pour le XXIè siècle: Mélanges en l'honneur de Samir Amin, sous la direction de Bernard Founou-Tchuigoua, Sams Dine Sy et Amady A. Dieng, l'Harmattan, Paris, 2003.
} 
GEORGES NZONGOLA-NTALAJA Les abordages théoriques sur les défis du développement des pays africains et les leçons qu'on peut en tirer

2. LeÇONS tiRÉES DU MOUVEMENT PANAFRICANISTE ET DE LA LUTTE POUR L'INDÉ́PENDANCE

Tandis que les Asiatiques ne se sont pas exprimés aussi vivement en opposition aux stratégies de développement occidentales que les Latino-Américains et les Africains l'ont fait à travers l'ECLA et la CEA, respectivement, ils ont été plus audacieux en rejetant ces stratégies en pratique. S'appuyant fortement sur des politiques interventionnistes, ils ont utilisé le pouvoir et les ressources de l'Etat pour industrialiser et, de surcroît, accroître la richesse et la prospérité de leurs pays respectifs. C'était aussi en Asie que la tendance autonomiste des pays du Sud par rapport aux grandes puissances mondiales se manifesta pour la première fois à la première conférence afro-asiatique tenue du 18 au 24 avril 1955 à Bandoeng, en Indonésie. Soucieux de définir pour eux-mêmes leurs plans de décolonisation, de développement économique et de paix, des leaders asiatiques et africains adoptèrent une position commune contre le colonialisme, le néocolonialisme, et la guerre froide, afin de promouvoir leurs intérêts légitimes pour l'autodétermination, l'indépendance économique, et un monde sans armes nucléaires.

Cet "esprit de Bandoeng" infusa un nouvel élan au projet panafricaniste vieil d'environ cinquante ans et dont Kwame Nkrumah du Ghana, Jomo Kenyatta du Kenya, et plusieurs autres militants étaient porteurs du message du 5è Congrès de Manchester (1945) faisant appel aux Africains de lutter pour l'autodétermination, l'indépendance économique, et la solidarité panafricaine. Pendant cette période de lutte pour l'indépendance, ce projet panafricaniste était compatible avec les aspirations des masses populaires qui, $\mathrm{d}$ 'après Amilcar Cabral, se battaient non pas pour des idées abstraites, mais pour la paix, les avantages matériels et un avenir meilleur pour leurs enfants. ${ }^{13}$ Comme Cabral, le distingué historien nigérian Jacob Ade Ajayi affirme que pour les masses africaines, la "notion de liberté n'était pas une abstraction, mais un catalogue de besoins spécifiques: libération de lois et directives injustes et incompréhensibles, restitution de leurs terres ... l'amélioration des conditions de vie en termes de logement et d'habillement, une meilleure récompense pour leurs efforts, des meilleures conditions d'exportation et de ventes de leurs produits agricoles, l'instruction en tant que facteur de mobilité sociale pour améliorer les conditions de vie de leurs enfants, et une provision adéquate d'eau potable,

13 Amilcar Cabral, Unité et lutte, Petite Collection Maspero, Paris, 1975, p. 292. 
d'électricité, de soins médicaux et d'autres nécessités de la vie". ${ }^{14}$

À cet égard, le plus grand défi du développement des pays africains est de pouvoir concevoir et exécuter des stratégies de développement susceptibles de satisfaire ces attentes de l'indépendance, qui correspondent encore aux aspirations profondes des masses africaines aujourd'hui. Pour ce faire, la classe dirigeante et architecte des politiques de développement devrait suivre les recommandations d'Amilcar Cabral en s'identifiant complètement et sans réservation aucune avec le peuple et ses aspirations, et non avec la bourgeoisie internationale et les politiques antisociales des institutions financières internationales sous son contrôle. ${ }^{15}$ C' est là le sens profond de l'analyse de Cabral de la transition du colonialisme à la période postcoloniale, que d'aucuns tendent à occulter en s'attardant un peu trop sur la notion de "suicide de classe", et courent ainsi le risque de réduire toute la politique économique et sociale de l'Etat indépendant à un moment fatidique pour la classe dirigeante.

Au-delà du choix de politique économique par la classe dirigeante, la question que pose Cabral est de surcroît plus fondamentale: un État indépendant basé sur le même système d'exploitation économique que l'État colonial, peut-il satisfaire les besoins des travailleurs et des paysans africains ? Répondant par la négative, il montre qu'il y a un choix à faire par la classe dirigeante, d'un côté entre le peuple et ses aspirations et, de l'autre, le système mondial et ses contraintes. C'est ainsi qu'il en appelle aux révolutionnaires africains à "détruire l'économie de l'ennemi et construire notre propre économie". ${ }^{16}$

Cette transformation de l'économie et de l'État hérités du colonialisme n'a pas eu lieu, la classe dirigeante ayant refusé la voie révolutionnaire, comme Frantz Fanon le montre bien, et opté par contre pour la voie facile d'enrichissement au sein des structures néocoloniales. ${ }^{17}$ Étant donné sa "volonté permanente d'identification" avec la bourgeoisie internationale ${ }^{18}$, la classe dirigeante africaine s'est contentée de jouer le rôle d'intermédiaire entre les centres domi-

14 Jacob Ade Ajayi, “Expectations of Independence”, Daedalus, 111(2): 5, (1982), passage traduit de l'anglais par Musifiky Mwanasali.

15 Cabral, Unité et lute, p. 148-170.

16 Ibid., p. 289-292.

17 Frantz Fanon, Les damnés de la terre, Petite Collection Maspero, Paris, 1970, p. 95-139.

18 Ibid., p. 118. 
GEORGES NZONGOLA-NTALAJA Les abordages théoriques sur les défis du développement des pays africains et les leçons qu'on peut en tirer

nants de l'économie mondiale et son peuple, et d'être rétribuée de ce fait. Les principales conséquences de cette option sont l'émergence d'une oligarchie africaine dont le penchant est l'utilisation du pouvoir d'Etat comme moyen d'enrichissement personnel, l'approfondissement du sous-développement du pays, et l'appauvrissement des masses populaires. Dans la plupart des cas, nous avons affaire non pas aux États développementalistes démocratiques dont le peuple a grandement besoin pour satisfaire ses aspirations, mais aux économies politiques de la prédation, au sujet desquelles Mbaya Kankwenda nous a livré une excellente analyse sur le Congo-Kinshasa. ${ }^{19}$

Pour s'opposer davantage au courant hégémonique de la théorie du développement, nous devons renouer avec le courant progressiste du mouvement de libération nationale, dans lequel on retrouve les premières formulations du courant contre-hégémonique tant dans le projet national africain d'autodétermination, d'indépendance économique et de solidarité africaine issu du panafricanisme que dans les idées d'Amilcar Cabral, Frantz Fanon, Patrice Lumumba et d'autres dirigeants de la lutte pour l'indépendance. À l'exhortation de Cabral aux intellectuels de s'identifier davantage avec le peuple auquel ils appartiennent, s'ajoutent les appels de Fanon d'aller à l'école du peuple pour mettre fin à l'aliénation de leur propre société et jeter les jalons d'une démocratie participative ${ }^{20}$, ainsi que le vœu de Lumumba que l'histoire africaine soit conçue et rédigée à partir d'une perspective africaine. ${ }^{21}$ Quant aux dirigeants qui ont malgré tout fait leur mieux pour la transformation économique et sociale de leurs pays respectifs, nous avons le devoir de reconnaître les efforts de Gamal Abdel Nasser pour l'industrialisation et le développement rural en Egypte, de Kwame Nkrumah pour la mise en place de l'infrastructure d'industrialisation au Ghana, de Julius Nyerere sur l'éducation et la construction nationale en Tanzanie, de Samora Machel pour développer le Mozambique et sa capacité de contribuer à la libération du Zimbabwe et de l'Afrique du

19 Mbaya J. Kankwenda, L'économie politique de la prédation au Congo-Kinshasa: Des origines à nos jours, 1885-2003, ICREDES, Kinshasa, Montréal et Washington, 2005.

20 Lire Rethinking Fanon: the continuing dialogue, sous la direction de Nigel C. Gibson, Humanity Books, Amherst, New York, 1999.

21 Vœu exprimé dans sa dernière lettre à son épouse, écrite dans la prison du camp militaire de Mbanza-Ngungu (ex-Thysville). Voir La pensée politique de Patrice Lumumba, sous la direction de Jean Van Lierde, Editions des Amis de Présence Africaine, Bruxelles, 1963. 
África (São Paulo, 1978, Online), São Paulo, n. 42, p. 156-176, 2021

Sud, et de Thomas Sankara pour renforcer les capacités des Burkinabés à se prendre en charge pour leur propre développement.

\section{LeÇONS tiRÉES DES CONFÉRENCES NATIONALES SOUVERAINES DES ANNÉES 1990}

Aussi intéressantes que les leçons tirées du panafricanisme et de la lutte pour l'indépendance sont celles provenant des conférences nationales souveraines (CNS) des années 1990, qui enrichissent les leçons décrites ci-haut et offrent de nouvelles pistes de réflexion sur le courant contre-hégémonique et ses alternatives favorables au bien-être des peuples d'Afrique. En ce qui concerne les défis du développement, les leçons les plus utiles en provenance de ces forums démocratiques peuvent se résumer en trois formes de lutte, contre (a) la dictature et l'autoritarisme; (b) la corruption et l'impunité; et (c) l'exclusion sociale et politique.

\section{LUTTE CONTRE LA DICTATURE ET L'AUTORITARISME}

La Conférence des Forces Vives de la Nation, tenue à Cotonou, au Bénin, entre le 19 et le 28 février 1990, inaugura la vague de conférences nationales souveraines qui défrayèrent la chronique à travers les pays d'Afrique francophone, entre 1990 et 1993. Le professeur Paulin Hountondji, premier conférencier à ce séminaire, est l'un des intellectuels béninois ayant apporté une importante contribution au succès de la conférence béninoise. Un des objectifs majeurs de ces assises nationales était de mettre fin au détournement du pouvoir d'État au profit d'un individu et de son entourage. L'antidote à ce mal ne pouvait être autre chose que le recouvrement de la souveraineté populaire. Le peuple, en tant que souverain primaire, entrait en conférence - elle-même définie comme "le peuple en conférence" - pour décider de son sort. La souveraineté de la conférence résidait dans le fait, d'une part, qu'elle constituait l'autorité suprême de l'État en cette période d'exception et, d'autre part, que ses décisions étaient impératives et opposables à tous.

Un des acquis principaux des CNS fut la consécration de l'État de droit et du droit à la résistance, ou l'obligation des citoyens de se soulever contre tout pouvoir illégal et contre la prise du pouvoir par la force ou d'autres 
GEORGES NZONGOLA-NTALAJA Les abordages théoriques sur les défis du développement des pays africains et les leçons qu'on peut en tirer

moyens illégaux. On retrouve ce droit dans pratiquement toutes les constitutions des pays ayant tenu une CNS, où il reste un principe constitutionnel incontournable. Par exemple, ce droit est reconnu dans l'article 64 de la Constitution de 2006 en République démocratique du Congo (RDC), 16 ans après la CNS. Il faut reconnaître, cependant, que bien que rares, des manifestations de révolte contre la dictature et l'usurpation du pouvoir ont eu lieu avant et après les conférences nationales. Les premières manifestations de ce genre eurent lieu à Khartoum, au Soudan, en 1964 contre le général Ibrahim Abboud et encore en 1985 contre le général Jaafar al-Niméiry. Avant le "printemps arabe" de 2010-2011 en Tunisie, Egypte et Lybie, la plus impressionnante manifestation de ce devoir civique en Afrique est sans aucun doute le soulèvement populaire du 25 octobre 2000 à Abidjan (Côte d'Ivoire), contre la tentative du général Robert Gueï de s'accrocher au pouvoir après avoir perdu l'élection présidentielle. La consolidation de la démocratie en Afrique aura besoin de ce pouvoir de la rue pour déstabiliser les dictateurs et dissuader les putschistes potentiels.

\section{LUTTE CONTRE LA CORRUPTION ET L'IMPUNITÉ}

La responsabilisation du peuple a également des implications salutaires dans la lutte contre la corruption et l'impunité. En tant que grande école de démocratie, la conférence nationale contribua énormément à l'éveil de la conscience nationale et du civisme, demandant à la population tout entière de ne plus tolérer la corruption et de dénoncer les coupables. À la CNS du Congo-Kinshasa, des renseignements sur les crimes économiques et politiques, y compris les assassinats, constituèrent les matières premières des commissions dites "sensibles", dont l'objet fut de faire toute la lumière sur les abus de pouvoir et les crimes odieux commis dans le passé. Ici, comme ailleurs, ce souci de transparence amena les conférenciers à faire le procès du régime sortant, avec des réquisitoires éloquents contre l'impunité et des plaidoyers en faveur de l'instauration d'un État de droit.

Avec une meilleure administration de la justice, l'État de droit représente une alternative heureuse aux régimes autoritaires. Or, cette alternative ne peut être porteuse de bonheur que dans la mesure où le processus de dé- 
mocratisation en cours se traduit en termes de gains sociaux appréciables et se consolide par la mise en application rigoureuse des principes d'égalité et d'équité. Par ailleurs, une meilleure prise en charge des fonctionnaires et des agents de l'ordre découragerait la petite corruption, une des causes principales des tracasseries administratives et policières. La grogne des fonctionnaires et d'autres catégories sociales dont le bien-être dépend, totalement ou en grande partie, de l'État est due à l'échec de ce dernier, dans bon nombre de pays, à assurer un paiement régulier des salaires, pensions et bourses d'études. Dans ces conditions, le recours à la corruption est sans doute lié à la pauvreté, aux charges sociales considérables et, généralement, aux difficultés que d'aucuns éprouvent à nouer les deux bouts.

Pour remédier à cette situation, une gouvernance démocratique, et particulièrement marquée par une meilleure gestion des finances publiques, est indispensable. Une fois les caisses publiques renflouées par des meilleures recettes fiscales, l'État sera mieux placé pour s'occuper des salaires et autres droits sociaux. Il disposera ainsi des ressources dont il a besoin pour mettre en œuvre sa stratégie économique de lutte contre la pauvreté, le chômage et l'exclusion sociale.

Cette lutte contre la corruption et pour la redistribution des ressources est partie intégrante du projet national basé sur les attentes populaires pour une vie meilleure, désignées communément comme les "dividendes de la démocratie". La contribution des CNS à la réalisation de ce projet réside dans les appels incessants au recouvrement du patrimoine national détourné par la corruption et les crimes apparentés, entre autres, la fuite des capitaux, les gaspillages associés à la construction des éléphants blancs, les dettes odieuses et les contrats léonins. La restitution aux peuples d'Afrique de biens mal acquis sous forme d'argent placé sur des comptes bancaires, en Bourse ou dans la propriété immobilière à l'étranger, est un droit imprescriptible pour ces peuples et leurs générations futures, tout en étant un devoir légal pour les pays du Nord. Comme receleurs de biens volés, ces pays sont aussi coupables que les voleurs africains: la bourgeoisie d'État décadente et les seigneurs de la guerre. 
GEORGES NZONGOLA-NTALAJA Les abordages théoriques sur les défis du développement des pays africains et les leçons qu'on peut en tirer

\section{LUTTE CONTRE L'EXCLUSION SOCIALE ET POLITIQUE}

Le développement économique implique le règlement de la question sociale par une attaque frontale contre la pauvreté, le chômage et les inégalités sociales, y compris celles entre hommes et femmes. Quant à la question nationale, sa résolution dans l'intérêt des peuples d'Afrique exige une lutte acharnée contre l'exclusion et l'impuissance politiques. C'est dire que toutes les composantes ethniques, culturelles et régionales de la communauté nationale doivent se sentir partie prenante dans la reconstruction de leur pays et la gestion de ses affaires. Faire en sorte que chacune d'elles ait voix au chapitre constitue la meilleure façon de prévenir des conflits violents.

Deux acquis majeurs des CNS, à cet égard, sont justement l'engagement solennel contre toute politique d'exclusion et son corollaire, la consécration du principe de partage du pouvoir. Malheureusement, la conception courante de ce principe se limite à la répartition des postes ministériels et autres hautes fonctions étatiques entre les élites appartenant aux groupes ethniques divers. C'est d'ailleurs cette logique qui anime l'agitation pour la multiplication d'entités provinciales et la préférence pour le fédéralisme comme mode d'organisation politico-administrative. Tout en tenant compte du souci légitime de respecter les équilibres régional, ethnique et de genre, la notion de partage du pouvoir qui répondrait aux aspirations populaires est beaucoup plus profonde. Elle concerne, en premier lieu, la géographie du pouvoir et des fonctions étatiques, lesquels doivent être décentralisés au profit des collectivités locales. La responsabilisation de ces dernières comme domaine privilégié de croissance et de développement économique implique la mise à leur disposition des moyens adéquats par une meilleure redistribution de recettes fiscales et une politique d'autonomie financière

En deuxième lieu, le principe de partage du pouvoir est dépourvu de sens dans l'absence d'une participation effective des classes populaires à la gestion de la chose publique. Le Brésil, ce pays qui a d'importants liens historiques et culturels avec l'Afrique, a la réputation d'être le berceau d'un modèle de démocratie qui réponde à cette exigence, avec le budget participatif inventé par la ville de Porto Alegre, qui permet aux habitants des différents quartiers de prendre part à l'affectation de fonds municipaux à leurs besoins 
de développement. ${ }^{22}$ Je n'ai jamais été à Porto Alegre, mais pendant ma carrière de sept ans comme expert en gouvernance au PNUD, une de mes tâches en matière de gouvernance locale était de faire connaître ce modèle de Porto Alegre à mes interlocuteurs au Nigéria et au Centre d'Oslo pour la gouvernance.

La démocratie à laquelle les peuples d'Afrique aspirent n'a rien à voir avec la démocratie libérale, dans laquelle la participation citoyenne se limite, le plus souvent, à la sélection de gouvernants parmi les représentants de la bourgeoisie et de familles illustres. Elle s'accorde beaucoup plus avec un système de démocratie participative et d'autogestion. Avec Claude Ake, nous affirmons que la démocratie dont l'Afrique a besoin est une démocratie sociale, ou celle "qui investisse davantage dans l'amélioration de la santé et l'éducation, ainsi que dans l'accroissement des capacités des peuples à participer effectivement". ${ }^{23}$ Une telle démocratie n'aura pas besoin de 50 ans d'expérimentation avec différentes stratégies de "réduction de la pauvreté", mais un programme d'attaque frontale contre la pauvreté et ses multiples racines, géré par ses propres bénéficiaires.

\section{Conclusion}

En clôturant ce séminaire international avec la discussion de deux approches contradictoires à l'analyse des défis du développement en Afrique, mon intention est d'interpeller les intellectuels africains et nos alliés en dehors du continent d'user de plus de créativité et de discernement dans leurs analyses des réalités africaines. J'en appelle à ces compagnons de lutte comme Kabengele Munanga, ses collègues de la CEA et tous les chercheurs qui ont les intérêts des masses africaines à cœur d'être plus innovateurs dans l'identification des alternatives favorables à ces dernières ainsi que des stratégies les plus appropriées pour leur matérialisation.

À l'heure du capitalisme triomphant, l'orthodoxie néolibérale a déjà proclamé la fin des idéologies, des théories alternatives, voire celle de l'histoire

22 Ignacio Ramonet, "Porto Alegre”, Le Monde Diplomatique, janvier 2001.

23 Claude Ake, Democracy and development in Africa, Brookings Institution, Washington, 1996, p. 132. 
GEORGES NZONGOLA-NTALAJA Les abordages théoriques sur les défis du développement des pays africains et les leçons qu'on peut en tirer

elle-même. Au lieu de contester cette affirmation dogmatique et de surcroît anti-démocratique de la pensée unique, nombreux sont des intellectuels africains qui font leur la profession de foi libérale et analysent les réalités de nos sociétés presque exclusivement dans leurs aspects empiriques au détriment des causes sous-jacentes, lesquelles sont de loin plus importantes. C'est bien ce genre de léthargie intellectuelle que le philosophe français Maurice Merleau-Ponty eut à déplorer dans une autre conjoncture dans sa leçon inaugurale au Collège de France en 1953. ${ }^{24}$ Pour lui, il était fort regrettable que des intellectuels se contentent de défendre sans le moindre sens critique toutes sortes de recettes, et de se plier à une orthodoxie quelconque. En tant que philosophes, ils devraient par contre s'impliquer dans une réflexion et une investigation sérieuses pour trouver quelque chose de nouveau.

Pour le sujet qui nous intéresse ici, il y a lieu de saluer tous ceux qui ont contribué et contribuent encore à la critique du courant hégémonique de la théorie du développement par un combat intellectuel générateur d'une meilleure compréhension des réalités africaines ainsi que des outils nécessaires pour résoudre les défis du développement auxquels les pays africains font face. Parmi ces combattants intellectuels, permettez-moi de rendre hommage, en ordre alphabétique, à Adebayo Adedeji, Claude Ake, Samir Amin, Amilcar Cabral, Frantz Fanon, Paulin Hountondji, Mbaya Kankwenda, Patrice Lumumba, Samora Machel, Gamal Abdel Nasser, Kwame Nkrumah, Julius Nyerere, Bade Onimode et Thomas Sankara.

Je vous remercie.

24 Maurice Merleau-Ponty, Éloge de la philosophie, Gallimard, Paris, 1953. 